\title{
GROWTH HORMONE LEVELS AT INDUCED HYPOGLYCEMIA; AN EFFECTIVE WAY OF DIAGNOSING GROWTH HORMONE DEFICIENCY
}

\author{
Nida Basharat, Asif Ali Memon, Sumbal Nida, Naveed Asif*, Saima Shakil Malik, Muhammad Tahir Khadim \\ Armed Forces Institute of Pathology/National University of Medical Sciences (NUMS) Rawalpindi Pakistan, *Combined Military Hospital \\ Quetta/National University of Medical Sciences (NUMS) Pakistan
}

\begin{abstract}
Objective: To introduce a relatively convenient and effective way of conducting Insulin Tolerance Test for diagnosis of Growth Hormone deficiency in children with short stature.

Study Design: Cross sectional analytical study.

Place and Duration of Study: Conducted at Department of Chemical Pathology and Endocrinology, Armed Forces Institute of Pathology, Rawalpindi, from May 2017 to Jul 2018.

Methodology: A total of 185 cases were included. Sample selection was done by non-probability consecutive sampling technique. Insulin tolerance test was performed by taking basal sample for serum growth hormone and plasma glucose levels before giving intravenous insulin bolus according to dose of $0.15 \mathrm{IU} / \mathrm{kg}$. Samples for Growth Hormone level were repeated at time of induced hypoglycemia (defined as plasma glucose level of $<2.8$ $\mathrm{mmol} / \mathrm{L}), 30$ minutes and 60 minutes post induction.

Results: Mean age of the patients was $10 \pm 4$ years, majority 120 (65\%) were males. In the study population, 41 (22\%) patients showed adequate response to insulin tolerance test while $144(78 \%)$ showed inadequate response. At level of induction, mean growth hormone levels were $31.9 \pm 18.8 \mathrm{mIU} / 1$ and $4.7 \pm 4.4 \mathrm{mIU} / 1$ in patients showing adequate and inadequate response respectively ( $p$-value $<0.05)$. Majority $32(78 \%)$ of the patients showing adequate response had peak growth hormone response $(>20 \mathrm{mIU} / \mathrm{l})$ at induction alone, followed by 30 minutes post induction; reflecting the significance of these two samples in diagnosis of growth hormone deficiency.

Conclusion: We concluded that there is a simpler and effective way of conducting insulin tolerance test based on two samples (induction and thirty minutes) which are sufficient for diagnosis of growth hormone deficiency.
\end{abstract}

Keywords: Growth hormone deficiency, Induced hypoglycemia, Insulin tolerance test, Serum growth hormone, Short stature.

This is an Open Access article distributed under the terms of the Creative Commons Attribution License (http://creativecommons.org/licenses/by/4.0), which permits unrestricted use, distribution, and reproduction in any medium, provided the original work is properly cited.

\section{INTRODUCTION}

Short stature is a social stigma and cause of concern among children and their parents. Short stature is defined as height that is two standard deviations below the mean height or approximately below third percentile for that age and gender ${ }^{1}$. Common causes of short stature include malnutrition, anemia, positive family history, hypothyroidism and constitutional delay of growth and puberty. Growth hormone $(\mathrm{GH})$ deficiency is still categorized as a rare cause of short stature. It may be idiopathic or pathological, familial or sporadic, isolated or linked to pituitary dysfunction $^{2}$. GH is usually the first hormone to be affected in case of any pathology involving pituitary;

Correspondence: Dr Nida Basharat, Chemical Pathology Dept, Armed Forces Institute of Pathology, Rawalpindi Pakistan Received: 25 Feb 2019; revised received: 22 Oct 2019; accepted: 06 Nov 2019 presence of additional pituitary hormone disorders reflecting severity of the disease ${ }^{3}$. The incidence of $\mathrm{GH}$ deficiency is estimated to be around 1:4000 to $1: 20000^{4}$ with GH deficiency being responsible for $14 \%$ cases of short stature in hospital setting 5 .

GH release from pituitary gland is of pulsatile nature with almost unmeasurable levels during daytime. As a result, a single basal GH level is not sufficient enough to discriminate $\mathrm{GH}$ deficient cases from healthy individuals ${ }^{6}$. The diagnosis of actual growth hormone deficiency is still a challenge and requires a multifaceted approach including auxological, biochemical, radiological and rarely genetic investigations. Growth velocity and degree of short stature are main considerations in deciding to continue evaluation ${ }^{7}$. All these confounding factors provided an 
impetus for introducing numerous pharmacological tests over the years to ease the diagnosis of GH deficiency but their reliability is still under question.Main reasons are lack of consensus on cut off values and normal age related references used, non-standardization of test protocols and variability among commercially available $\mathrm{GH}$ assays owing to molecular heterogeneity of $\mathrm{GH}$. Conventionally, atleast two separate provocative tests are recommended to assess the $\mathrm{GH}$ axis with normal GH peak response of $>10 \mathrm{ng} / \mathrm{ml}^{8}$. Diagnosis is further strengthened by evaluation of constant levels of circulating substances such as IGF-1 and its binding proteins.

ITT (Insulin tolerance test) is still considered as 'gold standard' for diagnosis of GH deficiency, despite being a full of risk and resource consuming procedure ${ }^{9}$. Worldwide, common practice for conducting ITT is to take at least four to five samples to monitor GH peak response. This practice of repeated sampling has made it a difficult and labour intensive procedure; not only for the patient but also for the pathologist. Despite the tiered approach for diagnosis of GH deficiency, pediatric endocrinologists still use ITT as the main investigatory tool for commencement of GH therapy in cases of short stature. This evokes a need to invent a more easy and practical procedure; yet maintaining its high sensitivity and specificity. Based on this protocol, we planned to investigate how predictive are various GH samples taken during ITT of clinical outcome and to assess the necessity of repeated sampling in such cases.

Purpose of this study was to devise a more practical and effective way of conducting ITT in a tertiary care setting. As the awareness and demand regarding $\mathrm{GH}$ provocative testing is increasing day by day as well as the number of short stature children visiting pediatric endocrinologists, it has become vital to strive towards a simpler approach for diagnosing GH deficiency. Although rare, a missed diagnosis is not affordable as GH therapy is highly efficacious in such cases. Study reviewed clinical procedures, identified challenges and provided key recommendations for best practice for management of growth disorders.

\section{METHODOLOGY}

A cross sectional analytical study was conducted at Endocrine Clinic at Armed Forces Institute of Pathology, Rawalpindi, to assess the current sampling protocol of Insulin tolerance test. Study was approved by Institution Review Board of AFIP (FC-CHP16-22/READ-IRB/18/ 906). A total of 185 patients were studied from May 2017 to July 2018. Sample size was calculated using WHO calculator, taking prevalence of growth hormone deficiency to be $14 \%$ in hospital settings ${ }^{5}$, with confidence interval of $95 \%$ and margin of error $5 \%$. Sample selection was done by non-probability consecutive sampling technique after taking informed consent from parents of each patient.

All patients below eighteen ${ }^{10}$ years of age, fulfilling the criteria of short stature (height lying $<3^{\text {rd }}$ centile on growth chart) and advised ITT were included in the study.

Patients with a definitive cause of short stature (hypothyroidism, celiac disease, anemia, steroid therapy) were excluded from the study. All those cases with history of epilepsy and heart disease were also excluded.

Height in $\mathrm{cm}$ and weight in $\mathrm{kg}$ were measured using digital weighing machine with attached stadiometer (KERN MPC 250K 100M version 1.3) KERN and Sohn $\mathrm{GmbH}$ with a reproducibility of $+0.2 \mathrm{~cm}$ and $+0.1 \mathrm{~kg}$ respectively. These were plotted on Centers for Disease Control and Prevention (CDC) charts and further categorized into centiles. Each patient was advised blood complete picture, X-Ray wrist for bone age, serum thyroid stimulating hormone (TSH) and anti-tissue transglutaminase antibody (anti- TTG) to rule out other causes of short stature like anemia, hypothyroidism and celiac disease. Majority of the patients had already been evaluated for growth hormone deficiency, either by exercise stimulation test or levodopa stimulation test and showed inadequate stimulation. 
Children and their parents were briefed regarding the risks and complications associated with ITT. Test was performed in the morning (8$10 \mathrm{am})$ after overnight fasting of at least 10 hours. Procedure was started after passing an indwelling heparin lock venous cannula for subsequent sampling. Basal sample was taken for GH and plasma glucose levels. Patients were given intravenous insulin (Regular Humulin) bolus according to dose of $0.15 \mathrm{IU} / \mathrm{kg}$. After administration of insulin, patients were intensively monitored for plasma glucose levels using glucometer every 5-10 minutes. Sampling for GH was repeated at time of induced hypoglycemia (defined as plasma glucose levels $<2.8 \mathrm{mmol} / \mathrm{L}$ or symptoms of hypoglycemia), followed by a sample 30 minutes and 60 minutes post induction. Patients were allowed oral refreshment immediately at induction to recover plasma glucose levels and were kept under observation for one to two hours. Hypoglycemia was reverted with $10 \%$ dextrose $(2 \mathrm{ml} /$ $\mathrm{kg}$ ) where required.

Samples for growth hormone and plasma glucose were taken in plain gel tube and sodium fluoride tube respectively. Serum growth hormone levels were analyzed on Immulite 2000 by fully automated random access two site Chemilluminescent enzyme labeled immunoassay (Siemens Healthcare Diagnostics Inc. NY, USA) with an inter assay and intra assay precision of $5.7 \%-6.1 \%$ and $5.3 \%-6.5 \%$ respectively, linearity of $120 \mathrm{mIU} / \mathrm{L}$ and detection limit of $0.03 \mathrm{mIU} / 1$. Analysis of glucose was done on fully automated discrete random access chemistry analyzer ADVIA 1800 (Siemens Healthcare Diagnostics Inc. NY, USA) by hexokinase catalyzed method. Manufacturer provided controls were run with each batch of all analytes for internal quality control. External quality control was assured by simultaneously analyzing samples from External Quality Assurance Services (EQAS) and Randox International Quality Assessment Scheme (RIQAS). Patients having peak GH response of $>20 \mathrm{mIU} / 1$ at any point after insulin administration were categorized as having adequate response $^{4,11,12}$.
Data was analyzed using SPSS version 24 . Qualitative data was expressed as frequency with percentages whereas quantitative data was showed as mean \pm standard deviation. Non parametric data was expressed as median and inter quartile range (IQR). Data was tested for normality using Kolmogorove Smirnov test and comparison between groups was performed by Kruskal Wallis test for nonparametric data. Statistical significance was defined as $p$-value $<0.05$.

\section{RESULTS}

Total sample size was 185 out of which 120 $(65 \%)$ were male and $65(35 \%)$ were female. Mean age of males was $10 \pm 4$ years and of females was $9.8 \pm 3.8$ years. Table-I shows the mean biochemical parameters involved in the study.

One hundred and twenty six (68\%) of our study population was from rural area. Anti TTG levels were performed to rule out celiac disease. In $6(3 \%)$ of the patients anti TTG was positive.

Table-I: Baseline characteristics of study population $(n=185)$.

\begin{tabular}{l|c}
\hline Study Variables & Mean \pm SD \\
\hline Age (years) & $10 \pm 4$ \\
\hline Weight $(\mathrm{Kg})$ & $23.9 \pm 8.7$ \\
\hline Height $(\mathrm{cm})$ & $120.5 \pm 20.8$ \\
\hline BMI & $22.4 \pm 0.38$ \\
\hline $\mathrm{Hb}(\mathrm{g} / \mathrm{dl})$ & $11.9 \pm 1.2$ \\
\hline $\mathrm{TSH}(\mathrm{mIU} / \mathrm{l})$ & $3.1 \pm 1.3$ \\
\hline Bone Age X-ray (years) & $8.3 \pm 3.8$ \\
\hline Cortisol $(\mathrm{nmol} / \mathrm{l})$ & $265(328.5-214.5)$ \\
\hline Glucose basal $(\mathrm{mmol} / \mathrm{L})$ & $4.2(4.6-3.8)$ \\
\hline Glucose at Induction $(\mathrm{mmol} / \mathrm{L})$ & $1.9(2.1-1.7)$ \\
\hline Glucose at $30 \mathrm{~min}(\mathrm{mmol} / \mathrm{L})$ & $5.5(6.8-4.3)$ \\
\hline Glucose at $60 \mathrm{~min}(\mathrm{mmol} / \mathrm{L})$ & $5.7(6.8-4.8)$ \\
\hline GH Basal $(\mathrm{mIU} / \mathrm{L})$ & $0.82(2.6-0.4)$ \\
\hline GH at induction $(\mathrm{mIU} / \mathrm{L})$ & $5.0(14.2-1.4)$ \\
\hline GH at $30 \mathrm{~min}(\mathrm{mIU} / \mathrm{L})$ & $2.7(6.2-0.8)$ \\
\hline GH at $60 \mathrm{~min}(\mathrm{mIU} / \mathrm{L})$ & $0.97(2.2-0.32)$ \\
\hline
\end{tabular}

Only $9(5 \%)$ patients in our study population showed symptomatic hypoglycemia. Forty one $(22 \%)$ patients showed adequate response to ITT and 144 (78\%) patients showed inadequate response, indicating GH deficiency. Therefore, GH deficiency was found to be more common cause of 
short stature diagnosed by ITT, as compared to normal variant of short stature. Among males, $26(22 \%)$ patients showed adequate response. Among females, 16 (25\%) patients showed adequate response. In patients showing adequate response to ITT, 32 (78\%) showed peak GH response (>20 $\mathrm{mIU} / \mathrm{l})$ at $\mathrm{GH}$ induction alone, $3(8 \%)$ at ponse $(p$-value $<0.05)$. Kolmo-gorove Smirnov test was applied to test the normality of data. After confirmation of non-normality of data, a nonparametric Kruskal Wallis test was applied to test the mean difference between various $\mathrm{GH}$ samples and results are given in table-II for patients showing adequate and inadequate responses.

Table-II: Comparison of GH levels using Kruskal Wallis test in patients showing adequate and inadequate responses $(\mathrm{n}=\mathbf{1 8 5})$.

\begin{tabular}{|c|c|c|c|c|c|}
\hline \multicolumn{3}{|c|}{ Adequate } & \multicolumn{2}{|c|}{ Inadequate } & \multirow[b]{2}{*}{$p$-value } \\
\hline & Median & $p$-value & & Median & \\
\hline GH Levels & & \multirow{5}{*}{$<0.001$} & GH Levels & & \multirow{5}{*}{$<0.001$} \\
\hline $0 \mathrm{mint}$ & 33 & & $0 \mathrm{mint}$ & 59 & \\
\hline 30 mint & 39 & & 30 mint & 53 & \\
\hline 60 mint & 40 & & 60 mint & 52 & \\
\hline induction & 40 & & induction & 52 & \\
\hline
\end{tabular}

thirty minutes post induction, $5(12 \%)$ at both induction and thirty minutes later and $1(2 \%)$ at both thirty and sixty minutes post induction. Figure shows line plot of GH response during various samples in ITT.

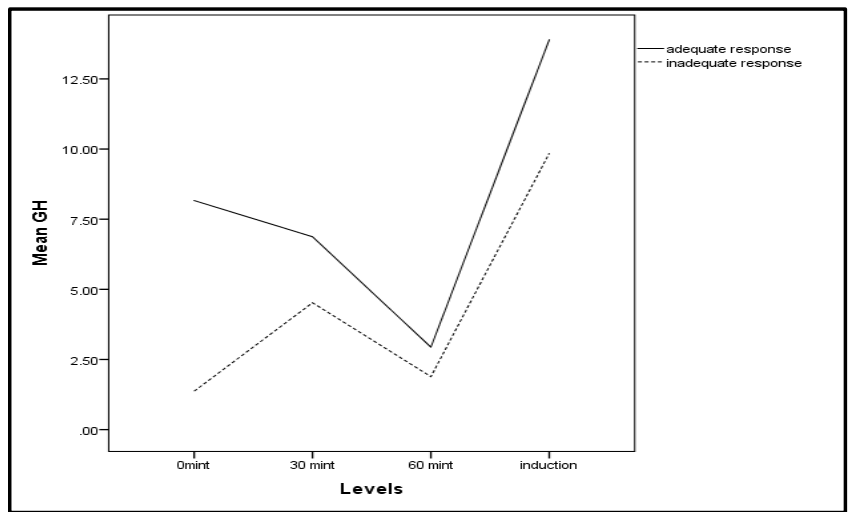

Figure: Line plot of mean $\mathrm{GH}$ values at different $\mathrm{GH}$ levels $(n=185)$.

At level of induction, mean GH levels were $31.9 \pm 18.8 \mathrm{mIU} / 1$ and $4.7 \pm 4.4 \mathrm{mIU} / 1$ in patients showing adequate and inadequate response respectively ( $p$-value $<0.05$ ). It was seen that maximum patients showed peak GH response at level of GH induction, followed by thirty minutes post induction; highlighting the significance of these two samples during ITT. Significant difference in $\mathrm{GH}$ levels amongst samples taken at induction, 30 minutes and 60 minutes was observed among patients showing adequate and inadequate res-

\section{DISCUSSION}

Purpose of our study was to evaluate the current sampling protocol followed for diagnosis of growth hormone deficiency using ITT in our set up. Current method for ITT is laborious and time consuming; involving a series of samples which might become difficult to execute, both for the patient and pathologist.

Few studies have analyzed the varying glycemic and GH excursions associated with ITT, showing a lower mean GH response $(5.4 \mathrm{mIU} / \mathrm{l})$ in $\mathrm{GH}$ deficient individuals as compared to normal individuals $(55.5 \mathrm{mIU} / \mathrm{l})^{2,13,14}$. In this study, we analyzed the methodology of periodic sampling in ITT based on glucose levels and GH levels and their effectiveness in diagnosis of $\mathrm{GH}$ deficiency. Result indicated that peak growth hormone responses which are used for ruling out GH deficiency mainly exist at induced hypoglycemia and thirty minutes post induction, which raises the question whether interpreting ITT should take into account the other two samples included in protocol.

Majority 144 (78\%) patients in our study were labeled GH deficient, which is in close proximity to a retrospective study conducted by Abdul moein et al. in 2011 in Jeddah15, where frequency of GH deficiency was around 69.4\% among 650 patients. Study showed female pre- 
ponderance which is contrary to current study. However, another study by Yousaf et al ${ }^{11}$ conducted among Pakistani population in 2016 showed majority $(66.6 \%)$ patients of short stature to be males, which is comparable to current study. Furthermore, another multi centric study by Sukran et al ${ }^{16}$ conducted in Turkey in 2015 involving 44 Turkish pediatric centres showed $50 \%$ of short stature children with normal growth velocity as $\mathrm{GH}$ deficient, using $\mathrm{GH}$ cut off of $30 \mathrm{mIU} / 1$ in ITT. Similar to current study, the most frequently used assay in this study was chemilluminescence immunoassay.

In another study conducted by Asif et al in Rawalpindi, Pakistan in 20095, frequency of GH deficiency based on ITT was around $60 \%$ which was also comparable to result of current study.

There is still ambiguity regarding the cut off values used for diagnosis of GH deficiency in provocative tests. Canadian Pediatric Endocrine group has defined a cut off of $24 \mathrm{mIU} / 1$ for diagnosis of GH deficiency using Immulite 2000. Meanwhile, most centers around UK use a cutoff of $21 \mathrm{mIU} / 1$ in $\mathrm{GH}$ stimulation tests ${ }^{17}$. In our study, we used $20 \mathrm{mIU} / 1$ as definitive cutoff, which was close to international studies. On the other hand Bonfig et al ${ }^{14}$ in 2008 stated the highest accuracy of ITT with a cutoff of $15 \mathrm{mIU} / 1$.

In another study conducted by Kevin et al in USA in 201218, which was based on glucagon stimulation test, it was seen that BMI and peak and nadir responses of glucose correlated negatively with GH levels. Our study was based on ITT but still reflected the same findings. Our study was not designed to determine specific $\mathrm{GH}$ cut points for ITT based on age and BMI, but does highlight the need for additional prospective studies based on control groups to provide definitive evidence in establishing cut off values.

Even though ITT remains the gold standard for diagnosis of GHD, there are not many studies to assess its reproducibility, reliability and sampling protocol to give the best clinical decision in case of short stature patients. Majority of short stature children may present with normal height velocity and show adequate response during ITT $^{12}$. Furthermore, there is still lack of consensus regarding the most appropriate $\mathrm{GH}$ assay available, with inter assay standardization and comparability as well as harmonization of cutoff values being the essential aspects to reduce variability ${ }^{19}$. Diagnosis of short stature requires a dynamic approach, especially while interpreting a blunted response to $\mathrm{GH}$ provocative tests in case of children presenting with normal growth rate. There is also a need to introduce new tests like GHRH plus arginine test to identify false GHD ${ }^{12}$. Diagnosis of short stature includes discrimination of normal growth variants like Constitutional Delay of Growth and Puberty (CDGP), familial short stature, Small for gestational age (SGA) with catch up growth from pathological growth impairment as both require different approach towards management. In our opinion, purpose of dynamic gold standard tests like ITT should be to identify those patients in need of GH replacement correctly, rather than identifying clinically insignificant defects. There are still grey areas in case of borderline cases, even after conducting ITT, so clinical judgement and follow up is still mainstay for assessment in such patients.

This study had certain limitations. Firstly, we did not include IGF-1 and IGF-BP3 levels in our diagnostic workup. Secondly, there is a need to conduct multi center studies before introducing the new strategy into clinical and laboratory practice.

\section{ACKNOWLEDGEMENT}

We acknowledge Ms Sumaira Mubarik for her useful contribution in statistical analysis.

\section{CONCLUSION}

We concluded that there is a simpler and convenient way of conducting ITT comprising of two samples (induction and thirty minutes post induction) which are sufficient for diagnosis of GH deficiency in case of short stature children. There is a need to inculcate this methodology in future diagnostic practice for the benefit of patient, treating physician and pathologist. 


\section{CONFLICT OF INTEREST}

This study has no conflict of interest to be declared by any author.

\section{REFERENCES}

1. John M, Koledova E, Kumar KM, Chaudhari H. Challenges in the diagnosis and management of growth hormone deficiency in India. Intl J Endocrinol 2016; 2016: 1-11.

2. Guo C, Chen L. Diagnostic value of provocative test by insulin combined with clonidine for growth hormone deficiency in children. Iranian J Pediatrics 2013; 23(3): 315-20.

3. Quigley CA, Zagar AJ, Liu CC, Brown DM, Huseman C, Levitsky L, et al. United States multicenter study of factors predicting the persistence of GH deficiency during the transition period between childhood and adulthood. Intl J Pediatric Endocrinol 2013; 2013(1): 6-13.

4. Wagner IV, Paetzold C, Gausche R, Vogel M, Körner A, Thiery J, et al. Clinical evidence-based cutoff limits for GH stimulation tests in children with a backup of results with reference to mass spectrometry. Europ J Endocrinol 2014; 171(3): 389-97.

5. Ali A, Hashim R, Khan FA, Sattar A, Ijaz A, Manzoor SM, et al. Evaluation of insulin-like growth factor- 1 and insulinlike growth factor binding protein-3 in diagnosis of growth hormone deficiency in short-stature children. J Ayub Med Coll 2009; 21(3): 40-45.

6. Dror N, Oren L, Pantanowitz M, Eliakim A, Nemet D. The Wingate anaerobic test cannot be used for the evaluation of growth hormone secretion in children with short stature. J Sport Health Sci 2017; 6(4): 443-46.

7. Stanley T. Diagnosis of growth hormone deficiency in childhood. Curr Opin Endocrinol Diabetes Obes 2012; 19(1): 47-52.

8. Rhee N, Oh KY, Yang EM, Kim CJ. Growth hormone responses to provocative tests in children with short stature. Chonnam Med J 2015; 51(1): 33-38.

9. Bozzola M, Meazza C. Growth hormone deficiency: diagnosis and therapy in children. Expert Rev Endocrinol Metab 2010; 5(2): $273-84$.

10. Cook DM, Rose SR. A review of guidelines for use of growth hormone in pediatric and transition patients. Pituitary 2012; 15(3): 301-10.

11. Yousaf S, Jaffar SR, Shaheen S, Shafiq F, Marium A, Raza S, et al. Growth hormone deficiency in short stature children diagnosed by insulin tolerance test in tertiary care hospital, Karachi, Pakistan. Pak J Pathol 2016; 27(4): 157-62.

12. Mazzola A, Meazza C, Travaglino P, Pagani S, Frattini D, Bozzola E, et al. Unreliability of classic provocative tests for the diagnosis of growth hormone deficiency. J Endocrinol Invest 2008; 31(2): 159-62.

13. Maghnie M, Aimaretti G, Bellone S, Bona G, Bellone J, Baldelli R, et al. Diagnosis of GH deficiency in the transition period: accuracy of insulin tolerance test and insulin-like growth factor-I measurement. European J Endocrinol 2005; 152(4): 589-96.

14. Bonfig W, Bechtold S, Bachmann S, Putzker S, Fuchs O, Pagel P, Schwarz HP. Reassessment of the optimal growth hormone cutoff level in insulin tolerance testing for growth hormone secretion in patients with childhood-onset growth hormone deficiency during transition to adulthood. J Pediatr Endocrinol Metab 2008; 21(11): 1049-56.

15. Eid Al- Agha A, Ocheltree A, Almalki MA, Sindi HM. Prevalence of growth hormone deficiency among short children at king abdul-aziz university hospital: a 5-year experience. Pak Pediatr J 2011; 35(2): 90-93.

16. Poyrazoğlu Ş, Akçay T, Arslanoğlu İ, Atabek ME, Atay Z, Berberoğlu $\mathrm{M}$, et al. Current practice in diagnosis and treatment of growth hormone deficiency in childhood. A survey from Turkey. J Clin Res Pediatr Endocrinol 2015; 7(1): 37-44.

17. Murray PG, Dattani MT, Clayton PE. Controversies in the diagnosis and management of growth hormone deficiency in childhood and adolescence. Arch Dis Child 2015; 101(1): 96-100.

18. Yuen KC, Biller BM, Katznelson L, Rhoads SA, Gurel MH, Chu $\mathrm{O}$, et al. Clinical characteristics, timing of peak responses and safety aspects of two dosing regimens of the glucagon stimulation test in evaluating growth hormone and cortisol secretion in adults. Pituitary 2013; 16(2): 220-30.

19. Junnila RK, Strasburger CJ, Bidlingmaier M. Pitfalls of insulinlike growth factor-i and growth hormone assays. Endocrinol. Metab Clin North Am 2015; 44(1): 27-34. 